ON THE PALLIO-TECTAL OR CORTICO-MESENCEPHALIC SYSTEM OF FIBRES.

BY CHARLES E. BEEVOR, M.D., F.R.C.P.,

AND

SIR VICTOR HORSLEY, F.R.S., F.R.C.S.

(From the Laboratory of Chemical Pathology, University College, Londor.)

\title{
Introduction AND METHOD.
}

IN the course of an investigation of the fibres composing the crus cerebri in a number of animals (twenty cats, eleven monkeys, one dog, and two badgers) localised lesions in the pallium or cortex cerebri bad been made by one of us (V. H.). While tracing the degenerations which resulted from such lesions we have specially followed out the connections which have for some years been known to exist between the cortex cerebri and the mesencephalon. In all cases the procedure was the same. A small area of the cortex in an animal anæsthetised with ether or chloroform was either excised or separated by a tangentially-directed incision through the corona radiata, so as to undermine the part determined upon.

Antiseptic precautions were always taken, and at the end of three weeks the animal was chloroformed, bled to death, and the vessels were washed out with Maller's fluid. The whole encephalon when fixed was cut into slabs, stained by Busch's modification of the Marchi method, and cut in serial sections. Only cases of healing by immediate union were accepted.

\section{Historr.}

The former investigations on this question may be grouped chronologically as follows :-

In 1891 the pathological observations of Zacher ${ }^{1}$ on man, 1 Archiv. fir Psych., Bd. xxii., 1891, p. 654. 
though necessarily indeterminate owing to the length of time elapsing between the occurrence of the lesion and the death of the patient, appeared to indicate that fibres pass from the occipital cortex to the mesencephalon by the lateral segment of the cerebral peduncle. His cases also suggest that the corpus geniculatum internum and the inferior corpus quadrigeminum were in connection by fibres with the cortex, and possibly with that of the temporal lobe.

In 1895 Edinger $^{1}$ first proved by the degeneration method in the bird the occurrence of an occipito-mesencephalic tract. The fibres proceeding from the occipital pole of the bird's hemisphere pass dorsally to the basalfrontal bundle and end in the deepest layers of the mesencephalon. The lesion was an extirpation of the "occipital" pole, and it extended forward to involve slightly the posterior part of the basal ganglia.

In the same year (1895) Boyce showed that after removel in the cat of the anterior third of one cerebral hemisphere there was in the middle region of the optic thalamus a group of scattered large degenerate fibres leaving the internal capsule and entering the optic thalamus, where they were grouped around the lower and inner aspect of the ventral lateral geniculate body. In more posteriorly-made sections they passed backward beneath the pulvinar and came to the surface as a well-marked group immediately external to the anterior corpus quadrigeminum. Some of these fibres entered the base of the corpus on the same side as the lesion, and probably some fibres terminated here; others he observed to cross over in the roof of the aqueduct to the opposite side, where they possibly terminated in either of the corpora quadrigemina. Fibres could also be traced back to the quadrigeminal region, being given off from the degenerate fibres in the capsule during their course to form the crusta. One well-marked bundle passed from the upper and incurved extremity of the pyramid just before the crusta is formed to the roof of the aqueduct, and lower down isolated tibres came off from the outer and upper extremity

' "Ueber die Entwickelung des Rindensehens," Archiv. fir Psych., Bd. xxvii., 1896, p. 950.

3 Phil. Trans. Roy. Soc., vol. 188, Series B, 1895, p. 895. 
of the crusta and curved round to the quadrigeminal region. Small groups of fibres left the dorsal and inner portion of the pes pedunculi and passed back to the tegmental commissural region of the same side, but whether they actually crossed over in the posterior commissure to the opposite side was not proved.

Lower down also he found that degenerated fibres left the crusta and passed back to the quadrigeminal region of the same side.

According to Dejerine ${ }^{1}$ the radiations of the anterior corpus quadrigeminum, i.e., tecto-pallial fibres, pass above and in front of the corpus geniculatum externum which they separate from the posterior segment of the internal capsule, and particularly from the bundle of Turck in front of the corpus geniculatum internum, and between this last and the pulvinar. Then they become superficial while running together to form the brachium of the anterior corpus quadrigeminum, and they spread out finslly into the superficial and middle layers of the anterior corpus quadrigeminum.

Simpson, ${ }^{9}$ in 1901, observed fibres in several cats in which " an attempt was made to divide the projection fibres arising from the left motor cortex in its whole extent." He observed fibres from the posterior aspect of the crusta passing back through the tegmentam and ending in the homolateral superior colliculus, ${ }^{3}$ a few crossing to the contra-lateral colliculus, while in addition a fine degeneration was also observed in the locus niger. In the dog and monkey he found no such fibres.

\section{Results of the Preshnt Regearch.}

\section{(1) General Statement.}

As the present research is founded chiefly on observations on the cat as representing the carnivora, and the macacque monkey (usually Macacus rhesus and more rarely Macacus

1 "Anatomie des Centres Nerveux," Tome ii., Fascicalus 1, 1901, p. 70.

2 Journal of Physiology, vol. xavil., 1901, p. 11.

3 The terms superior and inferior colliculus instead respectively of anterior and posterior corpus quadrigeminam, though not in general ase, appenr to deserve general adoption as being more sucoinct. 
sinicus) as representing the Primates, it will be understood that a general statement is only completely applicable to the higher of these animals, in which there is a well-developed non-excitable area (frontal) in front of the so-called motor or excitable cortex. With this proviso the general scheme of a pallio-tectal system may be stated as follows :-

From the frontal cortex no fibres could be traced by as to the mesencephalon; from the temporo-sphenoidal cortex very few fibres; while from the occipital lobe a fair number, and from the excitable cortex a large number of fibres can be followed to the corpora quadrigemina and mesencephalon.

The course taken by the different groups of fibres may now be detailed, and as the occipito-mesencephalic group is somewhat special by reason of the highly-developed visual association between the cortex of the caneal region and the superior colliculus (anterior corpus quadrigeminum) we will describe that first.

\section{(1) Occipito-Mesencephalic Fibres.}

The results we have obtained from the occipital lobe are strikingly concordant and fully confirm Edinger's original generalisations on this subject.

In proportion as more of the area of cortex containing Gennari's ${ }^{1}$ streak is involved in the lesion the number of degenerated fibres passing to the colliculns increases.

The occipital lesions produced in the present investigation were all situated on the outer surface of the pallinm.

In one observation in the monkey we made a small lesion involving the posterior lip of the "affen-spalte," to which Gennari's streak reaches in Macacus rhesus. From even this small lesion, some fibres passed to the anterior colliculus, showing how complete the association between the occipital area and the mesencephalon is.

The fibres which descend from the occipital cortex to the superior colliculus are large and stand out distinctly from the medium-sized occipito-thalamic and occipito-geniculate fibres and from the small callosal fibres and fine collaterals

- The comparative morphological topography of Gennari's streak is not yet worked out, but we have accumulated a large mass of material on whioh the statement above is based. 
which enter the corona radiata in large numbers. Some of the occipito-tectal fibres pass among the fibres composing Gratiolet's radiation, others run through the mesial region of the inferior longitudinal bundle (posterior fourth). This is in harmony with the converse observations of Schütz, made on microgyric brains, in which fibres of the inferior longitudinal bundle were found to be really tecto-occipital in character.

In traversing the thalamus the occipito-mesencephalic fibres run for the most part in the stratum zonale, but also partly through the lateral part of the dorsal nucleus.

A very few pass just mesially to the corpus geniculatum internum, and all are distributed to the whole breadth of stratum griseum profundum of the anterior colliculus.

\section{(2) Temporo-Mesencephalic Fibres.}

It will be better to divide the results from lesions of the temporal lobe, according to the animals, cat and monkey, operated on, because the homologies of the gyri in two so distant genera have not yet been accurately determined.

(a) Cat.-The terminology descriptive of the temporal gyri in the cat is best given in Flatau and Jacobsohn's ${ }^{1}$ handbook. We have made lesions in the small gyrus behind the Sylvian fissure, i.e., the gyrus Sylviacus posterior and in the next gyrus behind it, viz., the gyrus ecto-Sylvius posterior. Of these two, injury of the former only reveals fibres passing to the locus niger. Lesion of the posterior limb of the ecto-Sylvian gyras demonstrated an outflow of the fibres both large and small passing to the mesial side of the posterior extremity of the lenticular nucleus entering the outermost bundles of the internal capsule, and thence distributed to $(a)$ the optic tbalamus, $(b)$ the corpus geniculatum internum, $(c)$ the posterior brachium of the corpora quadrigemina (colliculi), (d) the pontime nucleus. Of these it is to be noted that a number of fibres in the posterior brachium turn up towards the posterior colliculus, but we have never been able to follow them into that ganglion; that

1 "Handbuch der Anatomio und Vergleichenden Anatomie des Centralnervensytems der Säugethiere." 1899. 


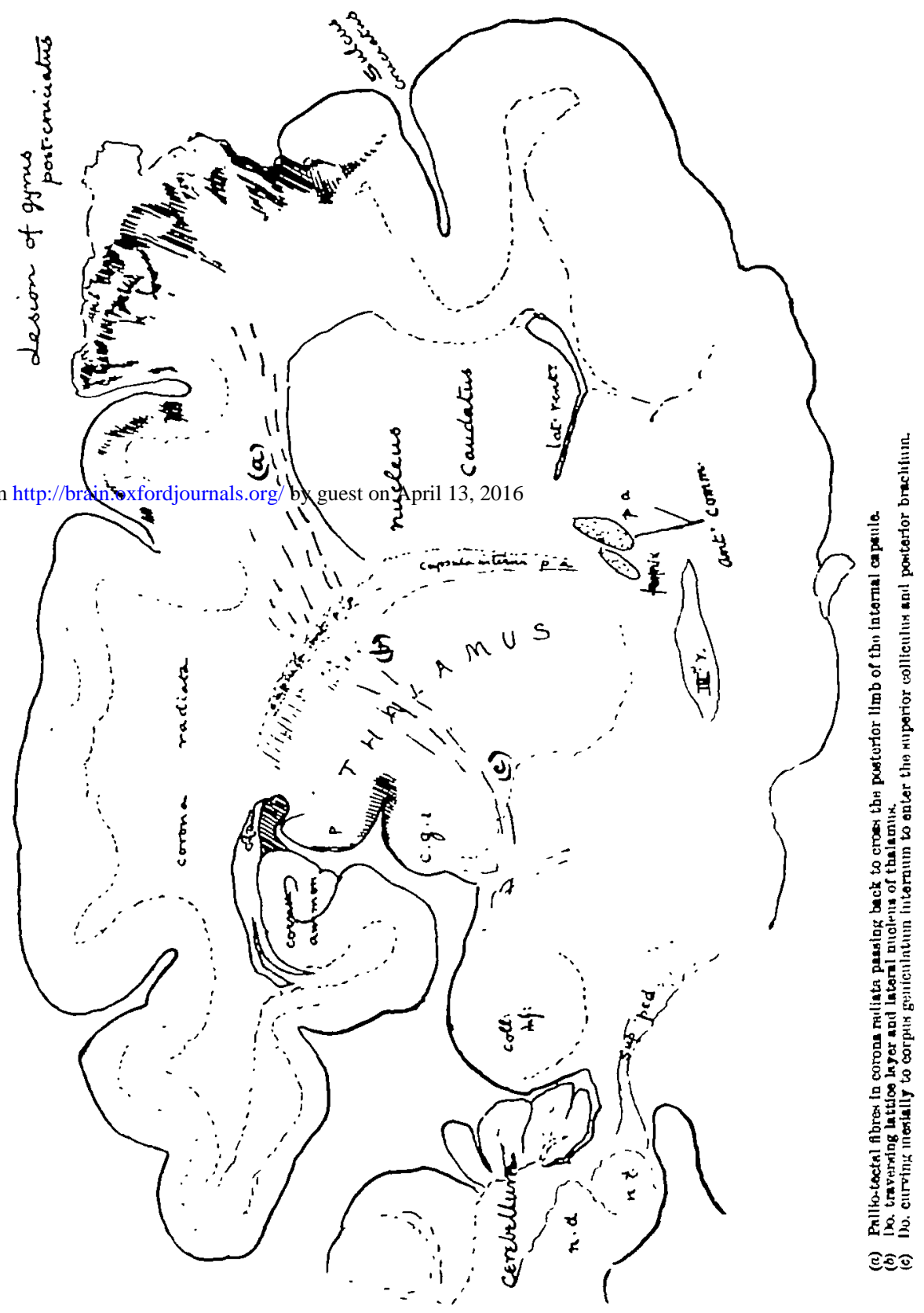




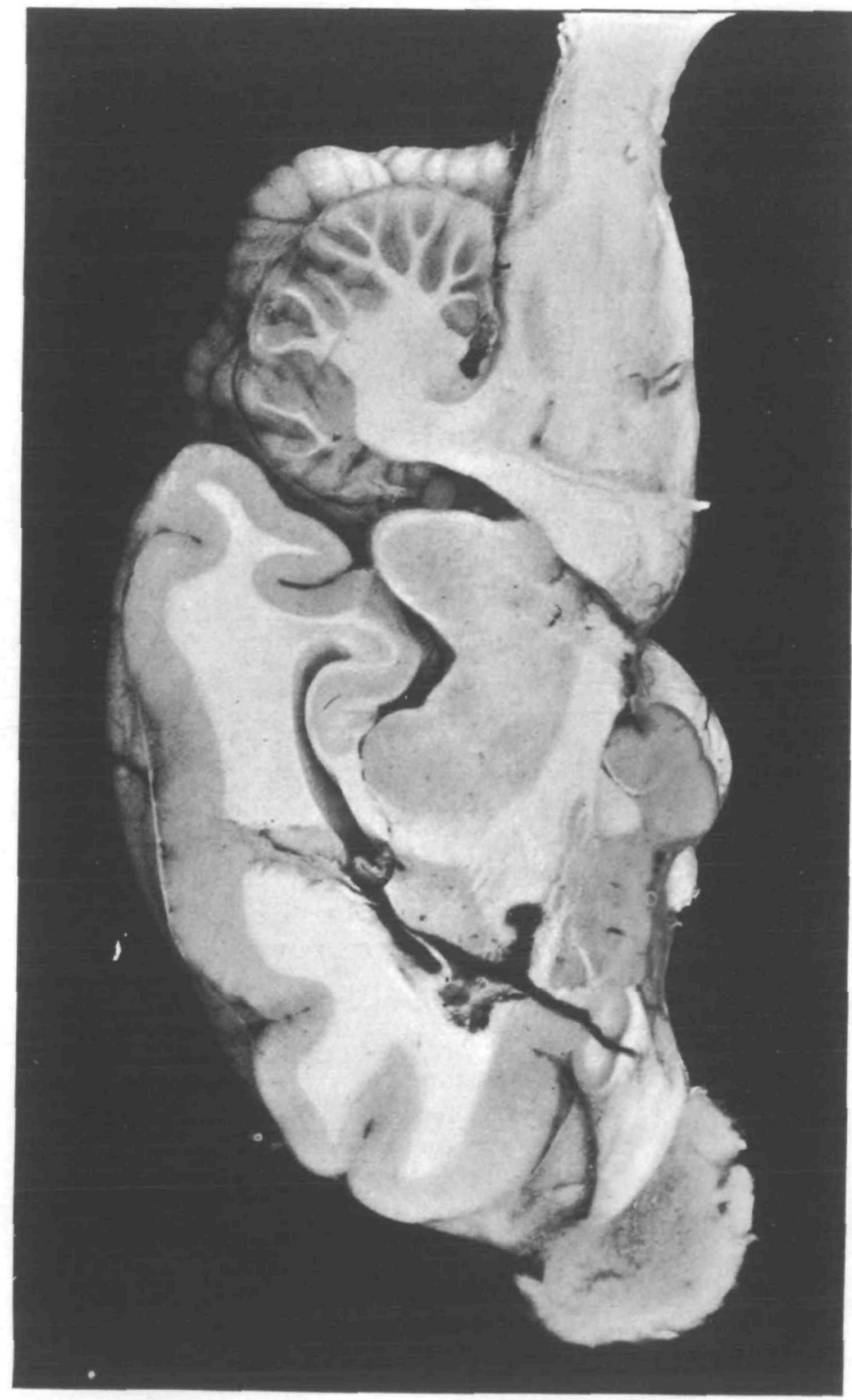

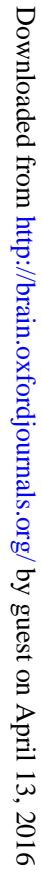




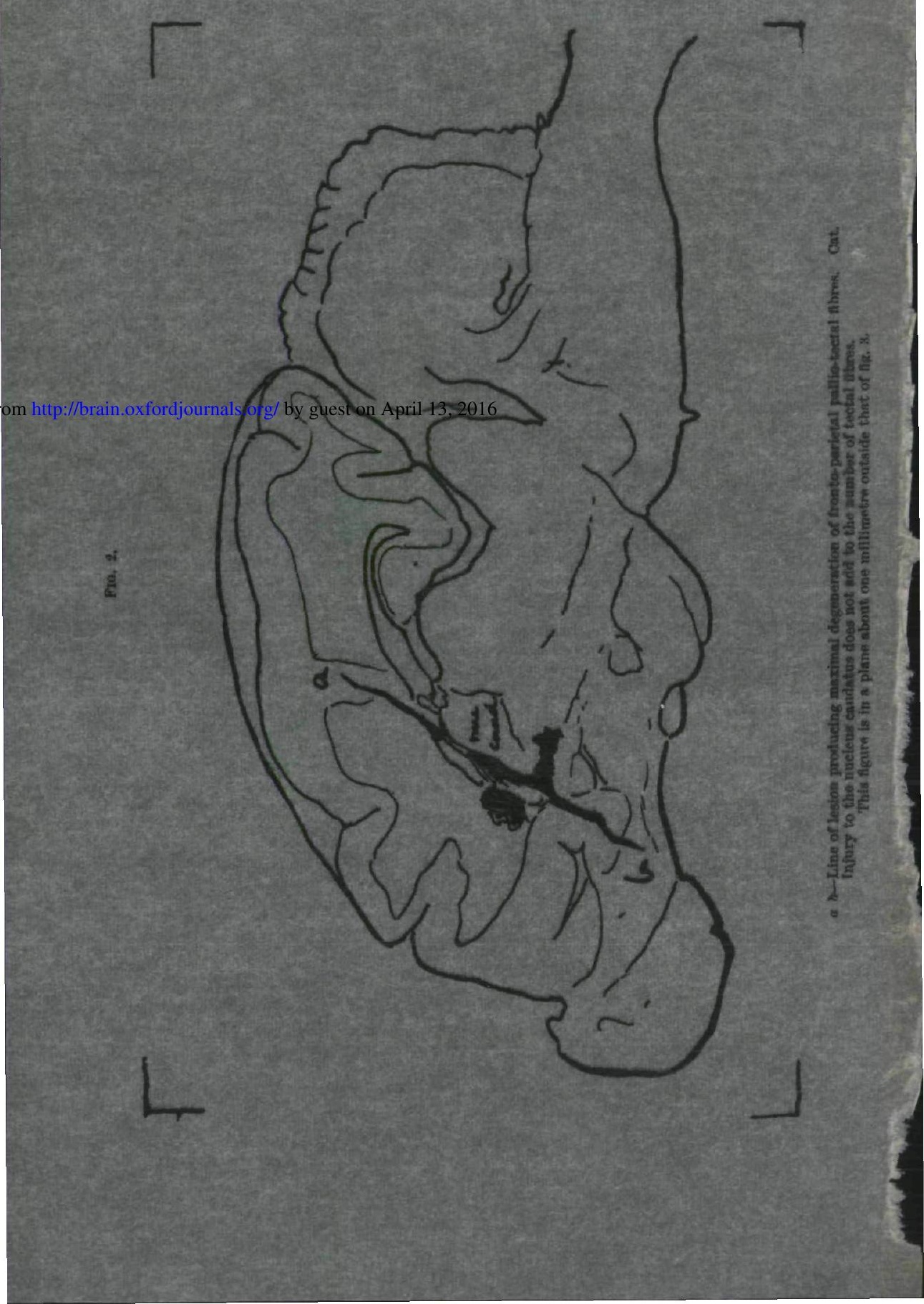




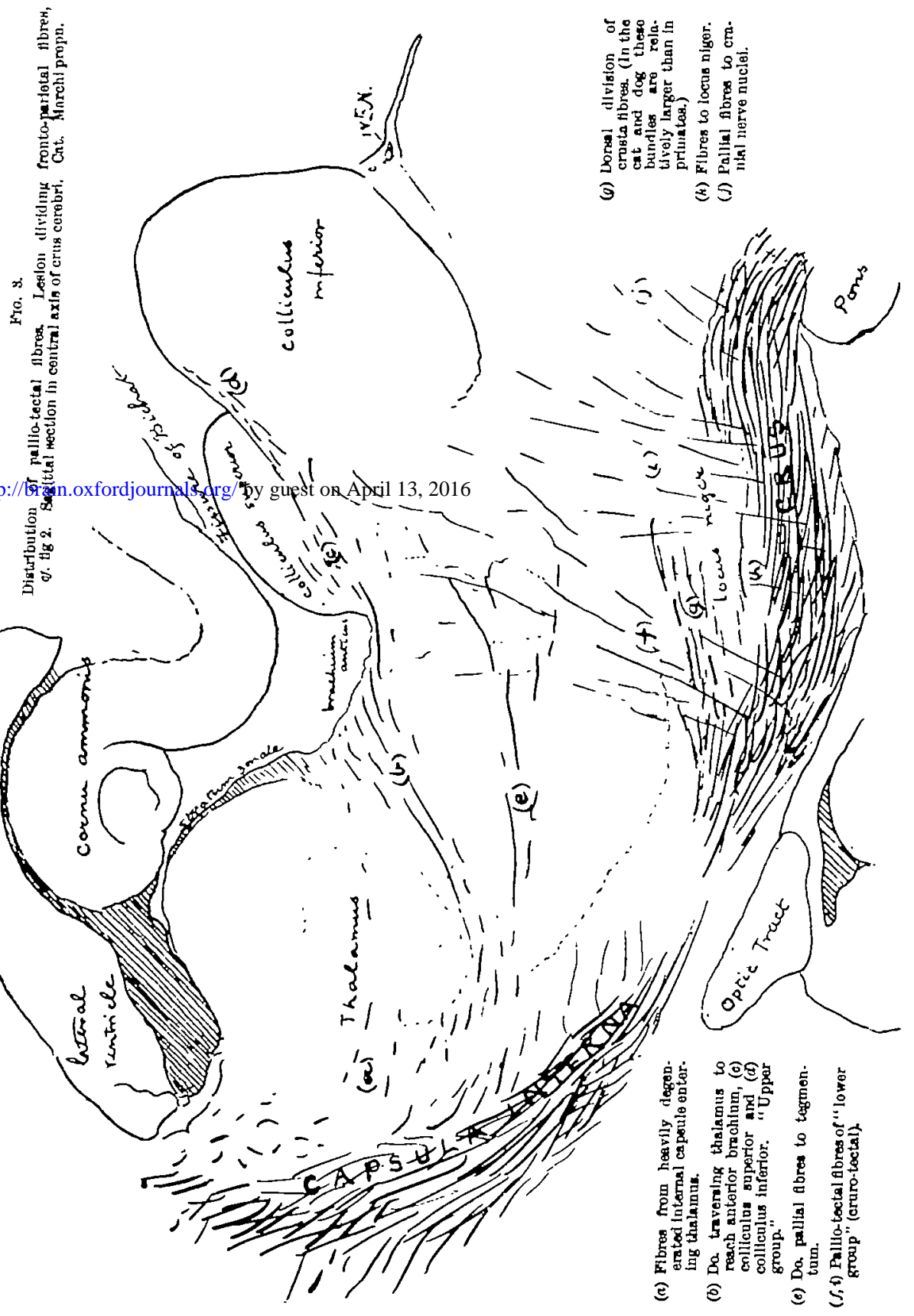




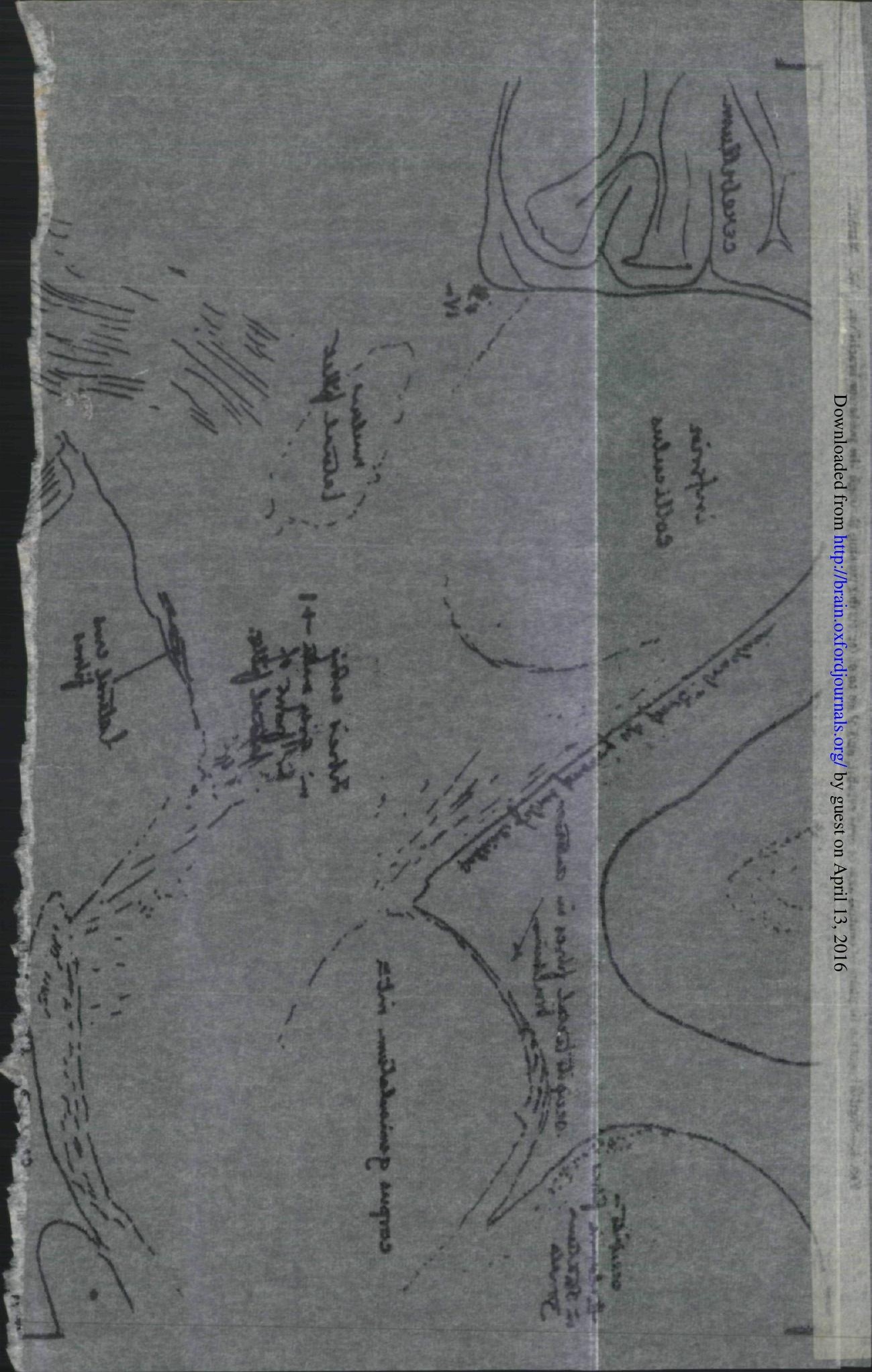




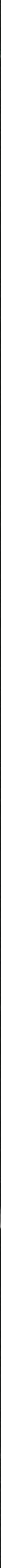


Cat Alawem pres.

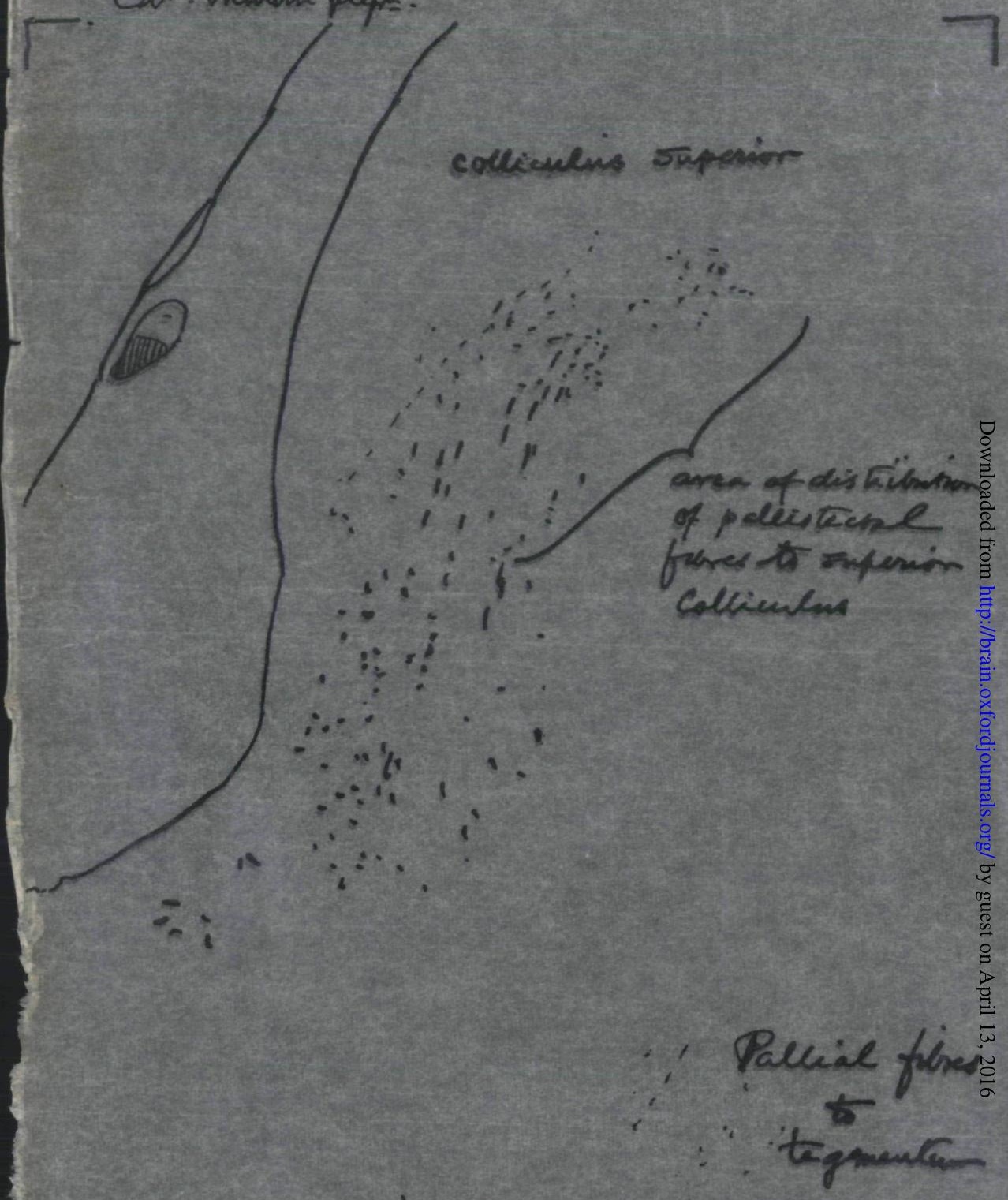

$\therefore$ palistectal fira

- crassiy here nitanal

- to mitemal geniculate

bry. 


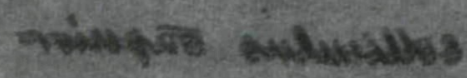

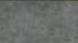

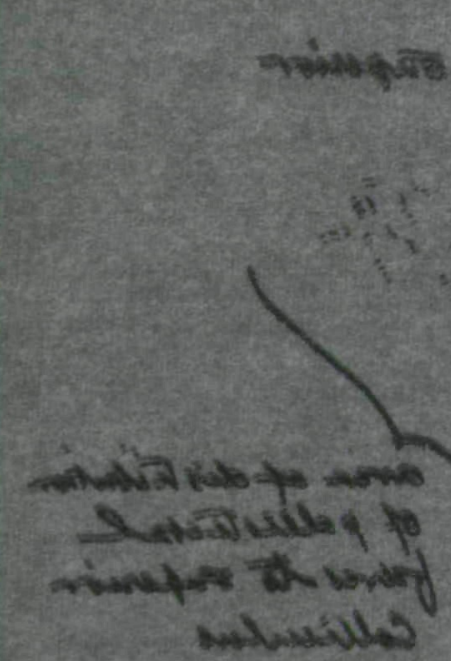




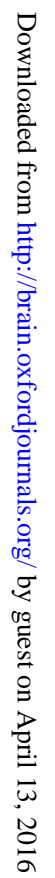


is, we have never been able to follow them beyond the gray Inatter forming the anterior fused border of the nucleus of the lateral fillet. The fibres which poss to the brachium are accompanied in the first part of their course by the fibres to the internal geniculate body. The fibres to the pons run down on the outside of the crus as Dejerine ${ }^{1}$ has shown in wan, and terminate in the upper and outer part of the frontal extremity of the pontine nucleus. In this animal (cat) they cannot be traced for a greater distance than onethird of the antero-posterior length of the pons.

(b) Monkey (Macacus rhesus).- - Of the lesions which we made in the temporal gyri, all involved the posterior half of the gyrus operated upon.

In all cases fibres were traced to $(a)$ the thalamus opticus, (b) the corpus geniculatum internum, (c) the posterior brachium, and $(d)$ the locus niger. These fibres for the most part are of fine or medium size, and they appear to be coarser in proportion as they arise from cortex which was nearer to the Sylvian fissure.

(3) Parieto-Mesencephalic and Combined Fronto- and Parieto-Mesencephalic Fibres.

The fibres coming from the excitable cortex we have investigated in two ways, either by producing a small lesion in a localised area of the so-called motor region, or by division of the corona radiata so as to undermine selected gyri, or by an extensive incision which was carried vertically through the hemisphere so as to separate the anterior third or balf more or less completely.

The results of these experiments will best be described as before, under the denomination of the animal investigated.

(a) Monkey.-In this animal the system of fibres connecting the excitable area with the mesencephalon is relatively only moderately developed, and was not in fact observed by Simpson in the two animals which be examined.

Lesions. - In two monkeys (Macacus sinicus and Macacus $r$ hesus) we made a horizontal incision through the hemisphere in the plane of the anterior end of the intraparietal

\footnotetext{
: "Anatomie des centres nerveur," Tome 2, Fascic. 1, p. 145.
} 
sulcus so as to cut off the fibres descending from the two central gyri. In a third animal (Macacus rhesus) we made a vertical stab incision beneath the whole length of the ascending parietal gyrus and continued beneath the two upper temporal gyri. In the two first cases fibres were traced to the thalamus opticas, but in one of them only could fibres be followed to the posterior brachium. In the third case, besides the fibres passing to the thalamus, others pass through the middle of the thalamus, some even entering the posterior commissure, while others were followed into the posterior brachium (probably temporo-mesencephalic fibres). A second group of fibres arise from the outer part of the crus and pass through the locus niger to ascend towards the tectum.

(b) Carnivora.-In three cats, one dog, and one badger, we made a strictly limited lesion in the post-crucial gyrus. In the one experiment on the dog we observed no fibres passing to the tectam (cf. Simpson loc. cit.), but this is only a single observation, and we do not therefore regard the question of their presence or absence in the dog as settled. In the cat and the badger a well-marked system of palliomesencephalic fibres exists. The fibres, for the most part large, leave the pyramidal tract in two defnite groups, which we may speak of as upper and lower respectively.

The upper group of fibres, which are apparently those previously seen by Boyce, leave the internal capsule at about the junction of its outer and middle thirds, as seen in horizontal section; they pass backwards through the thalamus to the inner side of the corpus geniculatum internum, partly enter the anterior colliculus, and partly pass beneath it to the posterior colliculus; some of these fibres seem to terminate in the thalamus.

The lower group, which we think might conveniently be also called the cruro-tectal group, arise from the crus cerebri at points all along the outer one-third of its dorsal surface, and the more anterior of these pass inwards and terminate in the anterior colliculus and locus niger, ${ }^{1}$ while the more

1 Probably these are the fibres traced by Sherrington and others as con. necting the crus and locus niger. 
posterior pass upwards and backwards to gain the posterior collicalus. Many of these latter run in the inferior brachium. These fibres to the posterior colliculus terminate in the anterior half of the large ovoid nucleus of that body.

A few of the outermost fibres turn down from among those which pass beneath the superior colliculus and run in or with the tractus transversus peduncularis.

Combined Frontal and Parieto-Mesencephalic Fibres.

In four cats we separated the whole fronto-parietal region of the hemisphere by an incision which passed through the junction of the anterior and middle thirds of the hemisphere.

The effect of this large division of the corona radiata was to increase the number of degenerated pallio-mesencephalic fibres, as well as to cause degeneration of large numbers of fibres terminating in the thalamus opticus. These latter observations, therefore, are confirmatory of the fact that the so-called motor region is in close association with the anterior colliculus.

\section{(4) Fronto-Mesencephalic Fibres.}

To examine the important question whether the frontal region, i.e., the area of cortex in front of the excitable cortex, sends any fibres to the mesencephalon we have made limited lesions of the frontal poles in two cats and frontal lobes in three monkeys. In the latter animal the posterior limit of the lesion was at least $4 \mathrm{~mm}$. distant from the nearest point from which movement resulted on excitation. Although we obtained striking degeneration of the fronto-thalamic fibres (Dejerine), in these cases the only mesencephalic centre to which we could follow fibres was the upper (i.e., anterior) part of the locus niger. No fibres, therefore, in our experience, go from the non-excitable part of the frontal lobe to the tectum. 